\title{
Health metrics in lung cancer care: Measure, improve, repeat
}

\author{
M. Shea Harrison, MD, C. Corbin Frye, MD, and Varun Puri, MD, MSCI
}

From the Division of Cardiothoracic Surgery, Washington University School of Medicine, St Louis, Mo. Disclosures: Authors have nothing to disclose with regard to commercial support.

Received for publication Oct 22, 2018; accepted for publication Oct 23, 2018; available ahead of print Nov 30 , 2018

Address for reprints: Varun Puri, MD, MSCI, 660 S Euclid Ave, Campus Box 8234, St Louis, MO 63110 (E-mail: varunpuri@wustl.edu).

J Thorac Cardiovasc Surg 2019;157:1236-7

0022-5223/\$0.00

Published by Elsevier Inc. on behalf of The American Association for Thoracic Surgery https://doi.org/10.1016/j.jtcvs.2018.10.104

With the nearly universal acceptance of a data-driven approach to improving health care outcomes, we have witnessed a rapid increase in the number of diseases or conditions for which care pathways are standardized. Accompanying this change, there is now significant focus on measuring adherence to the recommended care and correlating it with clinical and patient-reported outcomes. Not only has this approach been instrumental in creating standards of care, its importance has been recognized by many health care systems though public reporting of outcomes. Furthermore, efforts by payers to link quality of care to bundled payments and the downstream policies of health care systems to link provider compensation to adherence to established standards denote the significance of health metrics in the modern practice of medicine.

Care pathways and algorithms have historically been easier to establish in medical specialties. A key element contributing to this success has been the large body of high-quality randomized, controlled trials that have been conducted for common disease processes, such as diabetes and cardiovascular disease. Historically, resistance to such change in surgical disciplines has been related to the hierarchic nature of training and practice setups and to a wellestablished apprenticeship model that encourages the learning and propagation of "how I do it" rather than "why I do it" principles. With the emergence of such national bodies as the National Surgical Quality Improvement Project and the Surgical Outcomes Club of the American College of Surgeons, however, the surgical community is now understanding the value of a data-driven approach to surgery.

Surgical care is composed of 3 distinct phases: patient selection, performing the operation, and postoperative management. While it has been less controversial to develop and disseminate guidelines and quality measures for the preoperative (nutritional assessment, smoking cessation, etc) and postoperative periods (pain management, pulmonary toilet, etc), fewer efforts have been devoted to the development of intraoperative standards. A holistic

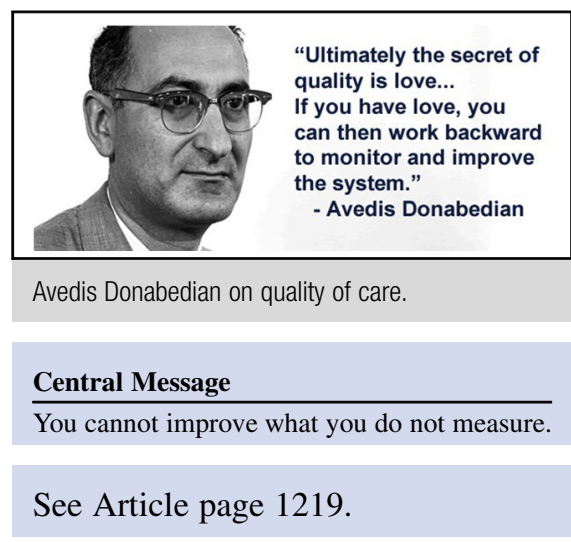

approach is especially important in lung cancer care, where multiple specialties are interacting in the management of complex patients with several comorbidities. In this light, the efforts by the Commission on Cancer to develop quality measures (QMs) for lung cancer and the work of Odell and colleagues ${ }^{1}$ to validate these standards, published in this issue of the Journal, are laudable.

Quality measures in the lung cancer surgical literature have been largely derived from evidence from smaller institutional studies and expert opinion. Several authors have validated proposed quality measures and suggested new measures by using collated data from administrative or claims databases. ${ }^{2-4}$ Samson and colleagues ${ }^{2}$ evaluated four QMs (anatomic resection, early operation within 8 weeks of diagnosis, achievement of negative surgical margins, and adequate sampling of 10 or more lymph nodes) and found that adherence to each additional QM improved survival in patients with stage 1 non-small cell lung cancer. ${ }^{2}$ Hudson and colleagues ${ }^{3}$ summarized the literature in the field and noted that several QMs were associated with improved survival: timely surgery in stage I cancer or within 4 months after neoadjuvant chemotherapy in locally advanced lung cancer, anatomic resection, adequate lymph node sampling, care by a multidisciplinary team, and adjuvant therapy in patients with pathologic stage higher than stage I.

Future improvements in guideline development for lung cancer surgery will require the collection of prospective data as well as patient-reported outcomes. Prospective databases, such as the Society of Thoracic Surgeons General Thoracic Database, are only partially suited because of their lack of long-term oncologic outcomes. Even more important is for the surgical community to embrace a culture of measuring, analyzing, and improving the care of our 
patients. Close attention to QMs is an ideal platform to promote this culture of high-quality care.

\section{References}

1. Odell DD, Feinglass J, Engelhardt K, Papastefan S, Meyerson SL, Bharat A. Evaluation of adherence to the Commission on Cancer lung cancer quality measures. $J$ Thorac Cardiovasc Surg. 2019;157:1219-35.
2. Samson P, Crabtree T, Broderick S, Kreisel D, Krupnick S, Patterson GA et al. Quality measures in clinical stage I non-small cell lung cancer: improved performance is associated with improved survival. Ann Thorac Surg. 2017;103:303-11.

3. Hudson J, Semenkovich T, Puri V. Oncologic quality indicators in thoracic surgery. Thorac Surg Clin. 2017;27:227-44.

4. Farjah F, Detterbeck FC. What is quality, and can we define it in lung cancer? - the case for quality improvement. Transl Lung Cancer Res. 2015;4:365-72. 